\title{
CIPROFLOXACINO ORAL DISCONTINUO EN RESECCIÓN TRANSURETRAL DE PRÓSTATA DE PACIENTES CON ORINA ESTÉRIL SIN USO DE CATÉTER PREOPERATORIO
}

Juan Pablo Valdevenito Sepúlveda y Enrique Hernández.

Servicio de Urología. Hospital Clínico Universidad de Chile. División de Bioestadística. Escuela de Salud Pública. Universidad de Chile. Santiago. Chile.

\begin{abstract}
Resumen.- OBJETIVO: Describir las complicaciones infecciosas con el uso de ciprofloxacino oral discontinuo en resección transuretral de próstata (RTU-P). Ponderar la influencia de los antecedentes clínicos, hallazgos y complicaciones intraoperatorias y evolución postoperatoria en el desarrollo de dichas complicaciones. Comparar los resultados con estudio previo de igual metodología en que se usó esquema antibiótico continuo hasta el retiro de la sonda uretrovesical.
\end{abstract}

MÉTODOS: Estudio prospectivo abierto de 105 pacientes consecutivos con orina estéril no usuarios de sonda uretrovesical, sometidos a RTU-P. Los pacientes recibieron ciprofloxacino $500 \mathrm{mg}$. oral (4 dosis) antes de su traslado a pabellón, la noche de la operación, la mañana siguiente de ésta y antes de retirar la sonda uretrovesical. Se excluyeron 5 pacientes después de su incorporación $(4,7 \%)$ y no hubo pérdidas en el seguimiento.

RESULTADOS: Se analizan 100 pacientes. Se presentó fiebre en el 10\% de los pacientes (temperatura axilar $\geq 37,5^{\circ} \mathrm{C}$. Se presentó infección clínica sistémica en el $3 \%$ de los pacientes (temperatura axilar $>38^{\circ} \mathrm{C}$ y proteína $C$ reactiva $>40 \mathrm{mg} / \mathrm{II}$. No se presentó bacteriuria postoperatoria aislada (recuento de colonias $>$ $10^{5} \mathrm{UFC} / \mathrm{mll}$. La presencia de prostatitis crónica activa se asoció estadísticamente con el desarrollo de fiebre $(p=0,018)$ y de infección clínica sistémica $(p=0,016)$. El antecedente de infección urinaria previa se asoció estadísticamente con la presencia de prostatitis crónica activa en la histopatología ( $p=0,049)$.

CONCLUSIONES: Este estudio muestra evidencia clínica de que el antecedente de infección urinaria previa es un factor de riesgo de complicaciones infecciosas en la resección transuretral de próstata y que gérmenes alojados en la próstata pueden ser responsables de ellas en este tipo de pacientes. El uso de una dosis antibiótica antes del retiro del catéter parece reducir la bacteriuria postoperatoria.

Palabras clave: Antibióticos. Resección transuretral de próstata. Prostatitis crónica.

Summary.- OBJECTIVES: To describe the rate of infectious complications using discontinuous oral ciprofloxacin in transurethral resection of the prostate. To weigh up the influence of clinical background, surgical complications and postoperative outcome on the development of such complications. To compare the results to those 
obtained will equal methodology using antibiotics until catheter removal.

METHODS: A prospective open study was designed including 105 consecutive patients with sterile urine and without indwelling catheter subjected to transurethral resection of the prostate. Patients received oral ciprofloxacin $500 \mathrm{mg}$ (4 doses) on call to the surgical room, the night of the surgery, next morning of surgery and before catheter removal.

RESULTS: One hundred patients are analyzed. Fever was present in 10\% patients laxillary temperature equal or over $37.5^{\circ} \mathrm{C}$. Systemic clinical infection was present in $3 \%$ patients laxillary temperature over $38^{\circ} \mathrm{C}$ and $\mathrm{C}$ reactive protein over $40 \mathrm{mg} / \mathrm{ll}$. No isolated postoperative bacteriuria was present (colony count $>10^{5} \mathrm{CFU}$ / $\mathrm{mll}$. Active chronic prostatitis was statistically asociated to fever $(p=0,018)$ and to systemic clinical infection $(p=0,016)$. Previous urinary tract infection antecedent was statistically associated to active chronic prostatitis on histopathology $(p=0,049)$.

CONCLUSIONS: This study shows some clinical evidence supporting that previous urinary tract infection antecedent is a risk factor of infectious complications after transurethral resection of the prostate and that prostate bearing microorganisms may be responsible for some of them in this kind of patients. An antibiotic dose before catheter removal seem to reduce postoperative bacteriuria.

Keywords: Antibiotics. Prostatic transurethral resection. Chronic prostatitis.

\section{INTRODUCCIÓN}

La septicemia sigue siendo una de las principales causas de muerte en la resección transuretral de próstata (RTU-P): Murphy y cols. reportan la muerte de 6 pacientes en 1.604 intervenciones $(0,34 \%), 4$ de las cuales fueron por sepsis (67\%); Mebust y cols. reportan 9 defunciones en 3.885 cirugías $(0,23 \%), 5$ de las cuales fueron por sepsis $(56 \%)(1,2)$.

Los principales factores de riesgo de complicaciones infecciosas de la RTU-P son la bacteriuria preoperatoria, el uso preoperatorio de sonda uretrovesical y los estados inmunodepresores. Se sabe que existen 2 momentos críticos para el desarrollo de complicaciones infecciosas graves después de una RTU-P: el periodo operatorio y postoperatorio inmediato y el día del retiro de la sonda uretrovesical (3).
En pacientes sin bacteriuria preoperatoria y no usuarios de sonda uretrovesical, el uso de antibióticos ha reducido la bacteriuria postoperatoria en promedio de un $26,5 \%$ a un $9,2 \%$ y la bacteriemia de un $4 \%$ a un $1 \%(4)$. La profilaxis antimicrobiana debe ser administrada antes de la cirugía y permitir un adecuado nivel tisular al momento de ésta, sin sobrepasar las 24 a $48 \mathrm{hrs}$. de uso (5). Sin embargo existe evidencia que hace razonable plantear el uso de antimicrobianos en RTU-P después de este período: un 2 a $10 \%$ de riesgo de desarrollar bacteriuria por cada día de uso de la sonda uretrovesical $(6,7)$ y el riesgo de bacteremia al retirarla $(1,8)$. La utilidad del uso de antibióticos más allá del retiro de la sonda Foley uretrovesical no estaría claramente demostrada (3).

En un estudio comparativo, prospectivo y aleatorio previo hemos demostrado una significativa menor frecuencia de infección urinaria postoperatoria (representada por una menor bacteriuria postoperatoria) con el uso de un esquema de Cefazolina 1 gr. intravenoso preoperatorio y cada $8 \mathrm{hrs}$. durante el primer día (3 dosis) seguido de Ciprofloxacino $250 \mathrm{mg}$. oral cada $12 \mathrm{hrs}$. hasta el retiro de la sonda uretrovesical, al comparar con el uso de un esquema de Cefazolina $1 \mathrm{gr}$. intravenoso preoperatorio y a las $8 \mathrm{hrs}$. postoperatorio (2 dosis) seguido de Nitrofurantoína $100 \mathrm{mg}$ oral en la noche hasta el retiro de la sonda (9). Sin embargo resulta claramente más sensato el uso de una droga única, la cual podría administrarse sólo en los momentos críticos de desarrollo de complicaciones infecciosas graves, en un afán no sólo de reducir el uso de antibióticos en estos pacientes, sino también reducir el desarrollo de resistencia antimicrobiana. Es por esto que planteamos un nuevo estudio en pacientes con orina estéril, no usuarios de sonda uretrovesical utilizando 4 dosis de Ciprofloxacino oral en forma discontinua, intentando a la vez ponderar la influencia de los antecedentes clínicos, hallazgos y complicaciones intraoperatorios y evolución postoperatoria en el desarrollo de dichas complicaciones. Estos resultados serán comparados con el primero de los esquemas antes descritos. Los resultados iniciales de este estudio fueron publicados previamente (10).

\section{MATERIAL Y MÉTODO}

Este es un estudio prospectivo abierto que incluyó pacientes con orina estéril, no usuarios de sonda Foley uretrovesical antes de la cirugía, sometidos a RTU-P entre marzo de 2004 y marzo de 2006. Se obtuvo consentimiento informado de todos los pacientes enrolados. Los criterios de exclusión fueron: 
a) historia de hipersensibilidad a ciprofloxacino;

b) uso de antimicrobianos 7 días antes de la cirugía;

c) Diabetes Mellitus insulino requirente;

d) uso crónico de corticoides o inmunosupresores;

e) usuarios de prótesis cardiacas y vasculares;

f) insuficiencia renal crónica (creatinina sérica mayor de $2,0 \mathrm{mg} / \mathrm{dl})$.

Cinco pacientes fueron excluidos después de su incorporación $(4,7 \%)$ : dos de ellos fueron finalmente sometidos a cirugía abierta, uno fue sometido sólo a una uretrotomía interna, uno era usuario de prótesis cardiaca y otro no tenía un cultivo de orina reciente.

Los pacientes recibieron 4 dosis de Ciprofloxacino $500 \mathrm{mg}$. oral (Grifociprox- $₫$, Laboratorio Chile SA): antes de su traslado a pabellón, la noche de la operación, la mañana siguiente de ésta y 1 hora antes del retiro de la sonda uretrovesical.

La cirugía se realizó bajo anestesia espinal o peridural; se aseptizó la piel con povidona yodada al $10 \%$, se utilizó Steri-Drape- $(3 \mathrm{M})$ para aislar escroto, periné y ano, y se usó como lubricante vaselina líquida estéril. La RTU-P se efectuó con resectoscopio Storz 24F sin flujo continuo utilizando solución de gli-

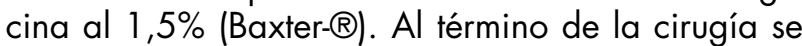
instaló sonda Foley uretrovesical de latex siliconado semirrígida 20 o $22 \mathrm{~F}$ (Porges- $(\Omega)$ la que se conectó a recolector urinario cerrado. En el postoperatorio se realizó irrigación vesical con solución salina al 0,9\% generalmente hasta el día previo al retiro de la sonda y en caso de obstrucción de ésta por coágulos se lavó con jeringa urológica usando igual solución.

En un protocolo estandarizado se consignaron los siguientes datos:

1) Características clínicas: edad, patologías asociadas, clasificación de la Sociedad Americana de Anestesia (ASA) (11) y los antecedentes de infección urinaria y biopsia prostática previas;

2) Parámetros quirúrgicos: tiempo operatorio, perforación capsular, secreción purulenta al corte del tejido y cirugía anexa transuretral;

3) Parámetros postoperatorios y complicaciones: número de lavados vesicales, necesidad de recambio de sonda Foley uretrovesical, necesidad de reoperación (revisión endoscópica), necesidad de transfusión sanguínea, días de uso de sonda Foley, días de hospitalización, y necesidad de rehospitalización y

4) Histopatología: gramos de tejido resecado, presencia de neoplasia intraepitelial prostática (NIP) o cáncer incidental y presencia de prostatitis crónica y prostatitis crónica activa (presencia de leucocitos polimorfonucleares).

Para la evaluación de las complicaciones infecciosas se registró cualquier alza térmica mayor de $37,{ }^{\circ} \mathrm{C}$ axilar. En caso de temperatura axilar igual o superior a los $38^{\circ} \mathrm{C}$ (fiebre alta) se solicitó proteína $C$ reactiva (PCR) asociada a urocultivo con recuento de colonias si el paciente se encontraba sin irrigación vesical (toma de muestra por punción de la sonda, con aplicación previa de antiséptico). La persistencia de fiebre alta (segundo día) o una PCR superior a $40 \mathrm{mg} / \mathrm{L}$ motivó toma de muestras para hemocultivos y el cambio de esquema antibiótico a Ciprofloxacino continuo o a otro esquema si no existía respuesta clínica. A todos los pacientes se les tomó cultivo de orina de muestra de orina de $2^{\circ}$ chorro entre las 2 y 3 semanas después del retiro de la sonda Foley uretrovesical.

Para el análisis de los resultados se consideraron como sucesos clínicos relevantes: 1) Fiebre (temperatura axilar de $37,5^{\circ} \mathrm{C}$ o superior); 2) Bacteriuria postoperatoria (recuento sobre 100.000 UFC por $\mathrm{ml}$ de orina) y 3) Infección clínica sistémica (dos días de temperatura axilar superior a $38^{\circ} \mathrm{C}$ y/o PCR mayor de $40 \mathrm{mg} / \mathrm{L}$ ) con o sin confirmación microbiológica.

\section{Análisis estadístico}

Para correlacionar cada característica clínica, parámetro quirúrgico, parámetro postoperatorio, complicación y resultado de la histopatología entre si y con los sucesos clínicos relevantes se usó la prueba de Chi-cuadrado o la prueba exacta de Fisher (si existía algún evento con menos de cinco casos, especificado). Además se calculó la razón de probabilidad (odds ratio) de aquellas asociaciones estadísticamente significativas. Para comparar el esquema antibiótico discontinuo y continuo se usó la prueba de Chi-cuadrado o la prueba exacta de Fisher en el caso de las variables ordinales, y la prueba de $t$ de Student en el caso de las variables numéricas. Los datos fueron ingresados en el programa Stata 8.1 (Stata Corporation, 2003) y la significación estadística se definió como un $p<0.05$.

\section{RESULTADOS}

Se analizan 100 pacientes con seguimiento completo, dentro de los cuales se incluyeron 9 pacientes en que se demostró violación del protocolo $(9 \%)$. De éstos, seis pacientes recibieron dosis extra (6\%): cuatro pacientes recibieron una dosis extra de antibiótico en la inducción anestésica (tres pacientes 
Cefazolina 1 gr y uno Ciprofloxacino 200 mg IV) y dos pacientes recibieron una dosis extra de Ciprofloxacino oral el segundo día postoperatorio. Tres pacientes recibieron dosis menor $(3 \%)$ : no recibieron la segunda dosis de Ciprofloxacino.

Las características clínicas de los pacientes, los parámetros quirúrgicos y la histopatología se

\section{TABLA I. CARACTERÍSTICAS CLIINICAS, PARÁMETROS} QUIRÚRGICOS E HISTOPATOLOGÍA.

\begin{tabular}{|c|c|}
\hline \multicolumn{2}{|l|}{ CARACTERÍSTICAS CLÍNICAS } \\
\hline Edad (años) & $64,6 \pm 7,6$ \\
\hline ASA & $37 \%$ \\
\hline 2 & $62 \%$ \\
\hline 3 & $1 \%$ \\
\hline Cáncer prostático & $6 \%$ \\
\hline Diabetes mellitus & $20 \%$ \\
\hline ITU previa & $18 \%$ \\
\hline Biopsia prostática previa & $18 \%$ \\
\hline Cirugía prostática previa & $6 \%$ \\
\hline \multicolumn{2}{|l|}{ PARÁMETROS QUIRÚRGICOS } \\
\hline Tiempo operatorio (minutos) & $44,9 \pm 14$ \\
\hline Perforación capsular & $16 \%$ \\
\hline Secreción purulenta al corte & $13 \%$ \\
\hline Cirugía anexa transuretral & $9 \%$ \\
\hline \multicolumn{2}{|l|}{ HITOPATOLOGÍA } \\
\hline Gramos de tejido resecado & $16,2 \pm 7,2$ \\
\hline NIP incidental & $5 / 94(5.3 \%)$ \\
\hline Cáncer incidental & $1 / 94(1.1 \%)$ \\
\hline Prostatitis crónica & $91 \%$ \\
\hline Prostatitis crónica activa & $26 \%$ \\
\hline
\end{tabular}

IASA: clasificación Sociedad Americana de Anestesia; ITU: infección del tracto urinario; NIP: neoplasia intraepitelial de próstata). muestran en la Tabla I. Veinte pacientes eran diabéticos y dieciocho tenían antecedente de ITU previa. Se reporta perforación de la cápsula prostática en 16 pacientes y secreción purulenta al corte del tejido prostático en 13.

Las cirugías anexas transuretrales fueron las siguientes: 5 uretrotomías endoscópicas, 2 resecciones transuretrales de tumor vesical superficial y 2 cistolitotomías. El peso promedio de tejido resecado fue 16,2 gramos; 91 pacientes presentaron algún grado de prostatitis crónica, 26 de los cuales tenían componente activo. Los parámetros postoperatorios, complicaciones y sucesos clínicos relevantes se muestran en la Tabla II. Nueve pacientes requirieron de lavado de la sonda Foley uretrovesical por obstrucción por coágulos; un paciente fue sometido a revisión endoscópica por hemovejiga, requiriendo además transfusión de 2 unidades de glóbulos rojos. No hubo mortalidad en la serie. Diez pacientes presentaron fiebre en algún momento de la evolución, la que se presentó después del primer día postoperatorio, periodo en que no existía cobertura antibiótica. Tres pacientes no diabéticos presentaron infección clínica sistémica (3\%): un paciente evolucionó con

TABLA II. PARÁMETROS POSTOPERATORIOS, COMPLICACIONES Y SUCESOS CLIINICOS RELEVANTES.

\section{PARÁMETROS POSTOPERATORIOS Y COMPLICACIONES}

\begin{tabular}{|ll|}
\hline Lavados vesicales & $9 \%$ \\
\hline Recambio de sonda Foley & $3 \%$ \\
\hline Revisión endoscópica & $1 \%$ \\
\hline Transfusión sanguínea & $1 \%$ \\
\hline Días de uso de sonda Foley & $3.75 \pm 0.89$ \\
\hline Días de hospitalización & $4.40 \pm 1.08$ \\
\hline Re-hospitalización & 0 \\
\hline & \\
\hline SUCESOS CLíNICOS RELEVANTES & \\
\hline Fiebre & $10 \%$ \\
\hline Infección clínica sistémica & $3 \%$ \\
\hline Bacteriuria postoperatoria aislada & 0 \\
\hline
\end{tabular}


obstrucción de la sonda uretrovesical por coágulos requiriendo seis lavados vesicales, recambio de ésta y finalmente revisión endoscópica por hemovejiga al $7^{\circ}$ día postoperatorio; presentó fiebre alta durante un total de cuatro días y PCR seriada de 89 y $190 \mathrm{mg} / \mathrm{L}$; su urocultivo demostró un Enterobacter cloacae sólo sensible a amikacina e imipenem, siendo tratado satisfactoriamente con el primero de los antibióticos ya nombrados.

Un segundo paciente, con antecedente de ITU previa 6 meses antes, presentó fiebre alta al $2^{\circ}$ y $3^{\circ}$ día postoperatorio con PCR de $69 \mathrm{mg} / \mathrm{L}$ y urocultivo negativo, siendo tratado con Ciprofloxacino oral con buena respuesta.

Un tercer paciente, también con antecedente de ITU previa tres semanas antes, presentó fiebre alta al $3^{\circ}$ y $4^{\circ}$ día postoperatorio con PCR de $106 \mathrm{mg} / \mathrm{L}$ y urocultivo negativo, siendo tratado satisfactoriamente con Ciprofloxacino oral. Los tres pacientes antes citados tuvieron hemocultivos negativos. No se presentó bacteriuria postoperatoria tardía en el resto de los pacientes ni se objetivó episodios de epididimitis aguda $o$ uretritis.

Se ponderó la influencia de las características clínicas, parámetros quirúrgicos y postoperatorios, complicaciones y resultados de la histopatología en el desarrollo de las complicaciones infecciosas. Se encontró asociación estadística entre prostatitis crónica activa y fiebre (prueba de Chi-cuadrado, $\mathrm{p}=$ 0,01 ; prueba exacta de Fisher, $p=0,018$ ) y entre prostatitis crónica activa e infección clínica sistémica (prueba de Chi-cuadrado, $p=0,003$; prueba exacta de Fisher, $p=0,016$ ). El antecedente de ITU previa se asoció estadísticamente a prostatitis crónica activa en la histopatología $(p=0,049)$ y casi a la fiebre postoperatoria (prueba de Chi-cuadrado, $p=0,056$; prueba exacta de Fisher, $p=0,077$ ) y al desarrollo de infección clínica sistémica (prueba de Chi-cuadrado, $p=0,026$; prueba exacta de Fisher, $p=0,083$ ). La Tabla III muestra las razones de probabilidad lodds ratio) de estas asociaciones clínicas.

\section{DISCUSIÓN}

Este es un estudio prospectivo de pacientes consecutivos con bajo riesgo de complicaciones infecciosas sometidos a RTU-P, en el cual se utiliza un esquema de Ciprofloxacino oral con dosis sólo en los momentos críticos de desarrollo de complicaciones infecciosas graves (discontinuo), en un afán de reducir al máximo el uso de antibióticos en esta cirugía.

Tiene a su favor que un porcentaje bajo de pacientes fue excluido después de la incorporación al estudio $(4,7 \%)$ y que todos los pacientes fueron evaluados al término de éste. Tiene la limitante de ser un estudio abierto por lo cual está expuesto a una mayor cantidad de sesgos. Fue diseñado usando un

TABLA III. RAZONES DE PROBABILIDAD (ODDS RATIOI DE LAS ASOCIACIONES CLÍNICAS.

\begin{tabular}{|lllll|}
\hline ASOCIACIONES CLíNICAS & $\begin{array}{l}\text { Prueba Chi- } \\
\text { cuadrado }\end{array}$ & $\begin{array}{l}\text { Prueba exacta } \\
\text { de Fisher }\end{array}$ & $\begin{array}{l}\text { Razón de } \\
\text { probabilidad }\end{array}$ & $\begin{array}{l}\text { Intervalo de } \\
\text { confianza 95\% }\end{array}$ \\
\hline $\begin{array}{l}\text { Prostatitis crónica activa - } \\
\text { Fiebre }\end{array}$ & 0.01 & 0.018 & 5.25 & $1.434-19.134$ \\
\hline $\begin{array}{l}\text { Prostatits crónica activa - } \\
\text { Infección clínica sistémica }\end{array}$ & 0.003 & 0.016 &. & $2.383-$. \\
\hline $\begin{array}{l}\text { ITU previa - } \\
\text { Prostatitis crónica activa }\end{array}$ & 0.049 & - & 2.84 & $1.005-8.093$ \\
\hline $\begin{array}{l}\text { ITU previa - Fiebre } \\
\text { ITU previa - } \\
\text { Infección clínica sistémica }\end{array}$ & 0.056 & 0.077 & 3.61 & $0.969-13.715$ \\
\hline
\end{tabular}

(ITU: infección del tracto urinario). 
criterio clínico donde la decisión de evaluación microbiológica durante su desarrollo (cultivos de orina y sangre) se basó en parámetros objetivos definidos con anterioridad. En este sentido, no se consideró la toma de un cultivo de orina en pabellón justo antes de la cirugía ya que el resultado de éste se conocería en forma inoportuna.

Los microorganismos que determinan complicaciones infecciosas después de RTU-P pueden ubicarse en la próstata o uretra e incluso en instrumental contaminado o líquidos de irrigación $(3,5,12,13)$. La etiología bacteriana de la prostatitis crónica se ha atribuido fundamentalmente a enterobacterias Gram negativas aunque también a Streptococcus faecalis (14). Por otro lado la flora microbiana uretral está compuesta fundamentalmente por bacterias Gram positivas, sin embargo también presenta enterobacterias a nivel distal en entre un 5 y un $11 \%$ de los hombres $(5,15)$. Estos mismos microorganismos son responsables de las infecciones después de RTU-P (12) y se han aislado en cultivos de tejido prostático resecado de un $13,5 \%$ de pacientes sin bacteriuria preoperatoria y no portadores de sonda Foley uretrovesical antes de la cirugía (16). Nuestro estudio presenta 3 pacientes con complicaciones infecciosas sistémicas. En uno de ellos se aisló un gérmen multiresistente intrahospitalario producto de la pérdida del circuito cerrado del drenaje urinario al lavarse la sonda uretrovesical obstruida por coágulos. En los otros dos casos, que presentaron fiebre alta y PCR elevada en los cuales no se aisló germen, la ausencia de lavados vesicales en su evolución y la buena respuesta clínica a Ciprofloxacino oral permiten plantear que su cuadro clínico se debió a microorganismos endógenos; ambos tenían el antecedente de ITU previa. La asociación estadística cruzada entre el antecedente de ITU previa, la infección clínica sistémica y la presencia de prostatitis crónica activa en la biopsia operatoria permitiría plantear que las compli-

TABLA IV. COMPARACIÓN DE ESQUEMA ANTIBIÓTICO DISCONTINUO Y CONTINUO.

\begin{tabular}{|l|l|l|l|l|}
\hline & $\begin{array}{l}\text { Total } \\
\mathbf{n}=145\end{array}$ & $\begin{array}{l}\text { Esquema } \\
\text { discontinuo (1) } \\
\mathbf{n}=100\end{array}$ & $\begin{array}{l}\text { Esquema } \\
\text { continuo (2) } \\
\mathbf{n}=\mathbf{4 5}\end{array}$ & Valor de p \\
\hline Edad (años) & $64,3(7,88)$ & $64,6(7,65)$ & $63,6(8,41)$ & 0,511 \\
\hline Diabetes mellitus & $22(15 \%)$ & $20(20 \%)$ & $2(4 \%)$ & 0,022 * \\
\hline ITU previa & $24(17 \%)$ & $18(18 \%)$ & $6(13 \%)$ & 0,484 \\
\hline Perforación capsular & $22(15 \%)$ & $16(16 \%)$ & $6(13 \%)$ & 0,679 \\
\hline Secreción purulenta al corte & $17(7 \%)$ & $13(13 \%)$ & $4(9 \%)$ & 0,585 * \\
\hline Lavados vesicales & $16(11 \%)$ & $9(9 \%)$ & $7(16 \%)$ & 0,244 \\
\hline Días de uso de sonda Foley & $3,8(0,96)$ & $3,75(0,89)$ & $3,93(1,11)$ & 0,292 \\
\hline Gramos de tejido resecado & $17,21(8,42)$ & $16,29(7,26)$ & $19,29(10,38)$ & 0,048 \\
\hline Prostatitis crónica activa & $35(24 \%)$ & $26(26 \%)$ & $9(20 \%)$ & 0,435 \\
\hline Fiebre & $11(7,6 \%)$ & $10(10 \%)$ & $1(2 \%)$ & 0,173 * \\
\hline Infección clínica sistémica & $3(2 \%)$ & $3(3 \%)$ & 0 & 0,552 * \\
\hline Bacteriuria postoperatoria aislada & $1(0,7 \%)$ & 0 & $1(2 \%)$ & 0,310 * \\
\hline
\end{tabular}

(1) Ciprofloxacino $500 \mathrm{mg}$ oral 4 dosis discontinuas

(2) Cefazolina $1 \mathrm{~g}$ intravenoso cada $8 \mathrm{~h}$ el $1^{\circ}$ día (3 dosis) seguido de Ciprofloxacino $250 \mathrm{mg}$ oral cada $12 \mathrm{~h}$ hasta el retiro de la sonda uretrovesical (Referencia 9)

(ITU: infección del tracto urinario)

* Prueba exacta de Fisher 
caciones infecciosas fueron causadas por gérmenes alojados en la próstata. Una posible causa es que aquellos pacientes con historia de ITU previa efectivamente tuvieran microrganismos en el tejido prostático en una mayor proporción y/o concentración, los cuales serían liberados al torrente sanguíneo durante la cirugía. Otra posibilidad es que dichos pacientes hayan estado con infección urinaria al momento de la cirugía, desarrollada después de la toma del último urocultivo: entre 1 y $6,5 \%$ de los pacientes que llegan a la intervención quirúrgica sin sonda uretrovesical supuestamente sin infección tienen urocultivo tomado en pabellón positivo (17-22). Se podría plantear que el antecedente de ITU previa hace menos aconsejable el uso de antibióticos en dosis discontinua en RTU$\mathrm{P}$; agregarlo como criterio de exclusión en próximos estudios, permitiría explorar esquemas antibióticos aun más reducidos en RTU-P de pacientes con bajo riesgo de complicaciones infecciosas.

Nuestro estudio muestra un 3\% de infección clínica sistémica lo que resulta ser alto en comparación con el 0,7 a $1 \%$ promedio de bacteriemia/septicemia descrito en pacientes sin bacteriuria preoperatoria sometidos a RTU-P con uso de antibióticos $(4,23)$. La definición no homogénea de esta complicación en los distintos estudios podría explicar la diferencia, así como también la alta frecuencia de prostatitis crónica activa en la biopsia de nuestros pacientes. La asociación entre el antecedente de ITU previa y el desarrollo de infección clínica sistémica con este esquema discontinuo es menor que la que informamos en nuestros resultados iniciales (prueba de Chi-cuadrado, $p=0,007$; prueba exacta de Fisher, $p=0,048)(10)$, pero seguimos creyendo que es un factor de riesgo real.

Se sabe que los protocolos antibióticos extendidos en RTU-P producen mayor disminución de la bacteriuria postoperatoria que aquellos de corta duración y de dosis única (23). Este estudio no muestra ningún caso de bacteriuria postoperatoria tardía, lo cual podría atribuirse a la dosis de antibiótico administrada antes del retiro de la sonda uretrovesical, anulando el riesgo inherente al uso por algunos días del catéter urinario $(6,7)$. Para aclarar este punto se debe diseñar un estudio aleatorio que específicamente compare el uso o no de esta dosis de antibiótico.

Finalmente si comparamos estos resultados con aquellos obtenidos con una similar metodología usando Cefazolina $1 \mathrm{gr}$ intravenoso cada 8 hrs durante el primer día (3 dosis) seguido de Ciprofloxacino oral $250 \mathrm{mg}$ cada $12 \mathrm{hrs} \mathrm{hasta} \mathrm{el} \mathrm{retiro} \mathrm{de} \mathrm{la} \mathrm{sonda}$ (9), no encontramos diferencias en el desarrollo de los sucesos clínicos relevantes (Tabla IV). Sin embargo se debe tener en cuenta que para comparar dos grupos que usan antibióticos, donde se pronostica una escasa diferencia en los resultados, los tamaños de muestra son pequeños (24).

\section{BIBLIOGRAFÍA Y LECTURAS RECOMENDADAS ( ${ }^{*}$ lectura de interés $y^{* *}$ lectura fundamental)}

1. MURPHY, D.M., FALKINER, F.R.; CARR, M. y cols.: "Septicemia after transurethral prostatectomy". Urology, 22: 133, 1983.

2. MEBUST, W.K.; HOLTGREWE, H.L.; COCKETT, A.T.K. y cols.: "Transurethral prostatectomy: immediate and postoperative complications. A cooperative study of 13 participating institutions evaluating 3,885 patients". J. Urol., 141: 243, 1989

*3. GRABE, M.: "Antimicrobial agents in transurethral prostatic resection”. J. Urol., 138: 245, 1987.

**4. QIANG, W.; JIANCHEN, W.; MACDONALD R. y cols.: "Antibiotic prophylaxis for transurethral prostatic resection in men with preoperative urine containing less than 100,000 bacteria per $\mathrm{ml}$ : a systematic review". J. Urol., 173: 1175, 2005.

*5. CHILDS, S.J.: "Appropriate surgical prophylaxis in transurethral genitourinary surgery and potential reduction in nosocomial infections". Urology, 27: 415, 1986.

6. KUNIN, C.M.; MCCORMACK, R.C.: "Prevention of catheter-induced urinary-tract infections by sterile closed drainage". New Eng. J Med, 274: $1155,1966$.

7. GARIBALDI, R.A.; BURKE, J.P.; BRITT, M.R. y cols.: "Meatal colonization and catheter-associated bacteriuria". New Eng. J. Med., 303: 316, 1980.

*8. DUCLOS, J.M.; LARROUTUROU, P.; SARKIS P.: "Timing of antibiotic prophylaxis with cefotaxime for prostatic resection: better in the operative period or at urethral catheter removal?". Am. J. Surg, 164: 21S, 1992.

9. VALDEVENITO SEPULVEDA, J.P.: “Antibióticos en resección transuretral de próstata de pacientes con bajo riesgo de complicaciones infecciosas: estudio comparativo prospectivo aleatorio". Arch. Esp. Urol., 57: 48, 2004.

10. VALDEVENITO, J.P.; VALDEVENITO, R.; GOMEZ, A. y cols.: "Ciprofloxacino oral discontinuo en reseccion transuretral de prostata. Resultados iniciales". Arch. Esp. Urol., 59: 274, 2006.

11. DRIPPS, R.D.; LAMONT, A.; ECKENHOFF, J.E.: "The role of anesthesia in surgical mortality". JAMA, 178: 261, 1961.

12. MADSEN, P.O.; LARSEN, E.H.; DØRFLINGER, T.: "The role of antibacterial prophylaxis in urologic surgery. Urology, 26: 38, 1985. 
13. LAST, P.M.; HARBISON, P.A.; MARSH, J.A.: "Bacteraemia after urological instrumentation. Lancet, 1: 74, 1966.

14. MEARES, J.R. EM.: "Prostatitis and related disorders. En: Walsh PC, Retik AB, Stamey TA, Vaughan Jr ED, eds. Campbell's Urology 6th ed. 807-22. W.B. Saunders Company, Philadelphia, 1992.

15. HELMHOLZ, H.F.: "Determination of the bacterial content of the urethra: a new method, with results of a study of 82 men". J. Urol., 64: 158, 1950.

*16. GORELICK, J.I.; SENTERFIT, L.B.; VAUGHAN, Jr. E.D.: "Quantitative bacterial tissue cultures from 209 prostatectomy specimens: findings and implications". J Urol, 139: 57, 1988.

17. SLAVIS, S.A.; MILLER, J.B.; GOLJI, H. y cols.: "Comparison of single-dose antibiotic prophylaxis in uncomplicated transurethral resection of the prostate". J. Urol, 147: 1303, 1992.

18. FERRIE, B.G.; SCOTT, R.: "Prophylactic cefuroxime in transurethral resection". Urol. Res., 12: 279, 1984.

19. MURDOCH, D.A.; BADENOCH, D.F.; GATCHALIAN, E.R.: "Oral ciprofloxacin as prophylaxis in transurethral resection of the prostate”. Br. J. Urol., 60: 153, 1987.

20. DESAI, K.M.; ABRAMS, P.H.; WHITE, L.O.: "A double-blind comparative trial of short-term orally administered enoxacin in the prevention of urinary infection after elective transurethral prostatectomy: a clinical and pharmacokinetic study". J. Urol., 139: 1232, 1988.

21. HARGREAVE, T.B.; BOTTO, H.; RIKKEN, G.H.J.M. y cols.: "European collaborative study of antibiotic prophylaxis for transurethral resection of the prostate". Eur. Urol., 23: 437, 1993.

22. VIITANEN, J.; TALJA, M.; JUSSILA, E. y cols.: "Randomized controlled study of chemoprophylaxis in transurethral prostatectomy". J. Urol., 150: 1715, 1993.

**23. BERRY, A.; BARRATT, A.: "Prophylatic antibiotic use in trnasurethral prostatic resection: a meta-analysis". J Urol, 167: 571, 2002.

24. FLETCHER, R.H.; FLETCHER, S.W.; WAGNER, E.H.: "Clinical Epidemiology: the essentials". 3rd ed. Williams \& Wilkins, Baltimore, 1996. 